\title{
Topological stability of a sequence of maps on a compact metric space
}

\author{
Dhaval Thakkar • Ruchi Das
}

Received: 26 April 2013 / Revised: 21 August 2013 / Accepted: 10 September 2013 / Published online: 22 September 2013

(C) The Author(s) 2013. This article is published with open access at SpringerLink.com

\begin{abstract}
In this paper we discuss the dynamical system induced by sequence of maps i.e. time varying map on a metric space. We define and study shadowing and expansiveness of such dynamical systems. We show that expansiveness and shadowing of time varying maps are conjugacy invariant. Finally, we prove that a time varying map having shadowing and expansiveness is topologically stable in the class of all time varying maps on a compact metric space.
\end{abstract}

Keywords Expansiveness $\cdot$ Shadowing $\cdot$ Conjugacy $\cdot$ Topological stability

Mathematics Subject Classification (2010) Primary 54H20; Secondary 37C75 . $37 \mathrm{C} 15$

\section{Introduction}

Expansiveness and shadowing are very important and useful dynamical properties of maps on metric spaces. They have lots of applications in Topological dynamics, Ergodic theory, Symbolic dynamics and related areas. One can refer [2,20] for detailed study of these notions. The concept of expansiveness originally introduced for homeomorphisms on metric spaces [24] has been generalized to positive expansiveness [9], point-wise expansiveness [21], entropy-expansiveness [3], continuum-wise expansiveness [13], measure expansiveness [17] and $n$-expansiveness [15]. Various

Communicated by S.K. Jain.

D. Thakkar · R. Das $(\varangle)$

Department of Mathematics, Faculty of Science,

The M. S. University of Baroda, Vadodara 390002, India

e-mail: rdasmsu@gmail.com 
kinds of shadowing also have been defined and their equivalences have been studied in $[20,14]$.

In [25], Walters has introduced the concept of topological stability and proved that Anosov diffeomorphisms are topologically stable. Expansiveness and shadowing play important role in the study of topological stability of maps on a compact metric space [26]. In [19], Nitecki has shown that topological stability is a necessary condition to get axiom A together with strong transversality. Morse-Smale flows are topologically stable is proved by Robinson in [22]. In [10-12], Hurley has obtained necessary conditions for topological stability. Moriyasu [16] has proved that the $C^{1}$-interior of the set of all topologically stable diffeomorphisms is characterized as the set of all $C^{1}$-structurally stable diffeomorphisms. In [18], authors have proved that, if $X^{t}$ is a flow in the $C^{1}$-interior of the set of topologically stable flows, then $X^{t}$ satisfies the Axiom A and the strong transversality condition. In [5], authors have proved similar result for the class of incompressible flows and also for volume-preserving diffeomorphisms. In $[4,5]$, authors have generalized results of $[5,18]$ for symplectomorphisms. Recently in [8], authors have studied expansiveness, shadowing, topological stability and decomposition theorems for homeomorphisms on non-compact and nonmetrizable spaces.

In discrete dynamical system $(X, f)$, where $X$ is a metric space and $f: X \rightarrow X$ is a continuous map, we consider the iterates of points of $X$ under the action of $f$ with discrete ticks of time. Here we consider the case when the function $f$ is changing with the ticks of time. i.e. we consider the action of sequence of functions $\left\{f_{n}\right\}_{n=0}^{\infty}$, where we always consider $f_{0}$ to be the identity map. We denote this action by $F$ and call $(X, F)$ a time varying dynamical system. For example, any moving picture on a television screen is an example of time varying dynamical system. In fact television screen is divided into pixels each of a single color red,blue or green. Also if $(X, \sigma)$ is a shift-space and $\left\{t_{n}\right\}$ is a sequence of integers then $\left\{\sigma^{t_{n}}\right\}$ is a time varying map on $X$. Similar kind of study related to random perturbations of dynamical systems has been done by Araújo [1]. In [23], introducing many new concepts authors have defined and studied chaos of a time varying map. i.e. of a time varying dynamical system. In [7], author has studied $G$-chaos of a sequence of maps in a metric $G$-space.

In this paper, we extend the notion of expansiveness and shadowing for time varying maps on a metric space and study them in detail. In Sect. 2, we define and study expansiveness of a time-varying map on a metric space. In Sect. 3, we define and study shadowing or P.O.T.P. for a time varying map on a metric space. In Sect. 4, we study topological stability of a time varying map on a compact metric space.

\section{Expansiveness of a time varying map}

Throughout we consider $(X, d)$ to be a metric space and $f_{n}: X \rightarrow X$ to be a sequence of continuous maps, $n=0,1,2, \ldots$ and $F=\left\{f_{n}\right\}_{n=0}^{\infty}$ be a time varying map on $X$. We denote

$$
F_{n}=f_{n} \circ f_{n-1} \circ \cdots \circ f_{1} \circ f_{0}, \text { for all } n=0,1,2 \ldots
$$


For any $i \leq j$ we define

$$
F_{[i, j]}=f_{j} \circ f_{j-1} \circ \cdots \circ f_{i+1} \circ f_{i}
$$

and for $i>j$, we define $F_{[i, j]}$ to be the identity map on $X$. For any $k>0$, we define a time varying map $\left(k^{t h}\right.$-iterate of $\left.F\right) F^{k}=\left\{g_{n}\right\}_{n=0}^{\infty}$ on $X$, where

$$
g_{n}=f_{n k} \circ f_{(n-1) k+k-1} \circ \cdots \circ f_{(n-1) k+2} \circ f_{(n-1) k+1} \text { for all } n \geq 0 \text {. }
$$

Thus $F^{k}=\left\{F_{[(n-1) k+1, n k]}\right\}_{n=0}^{\infty}$.

Definition 2.1 [23] Let $(X, d)$ be a metric space and $f_{n}: X \rightarrow X$ be a sequence of maps, $n=0,1,2, \ldots$ For a point $x_{0} \in X$, define a sequence as follows:

$$
x_{n+1}=f_{n+1}\left(x_{n}\right), \quad n=0,1,2, \ldots
$$

Then the sequence $O\left(x_{0}\right)=\left\{x_{n}\right\}_{n=0}^{\infty}$ is said to be the orbit of $x_{0}$ under time varying map (or sequence of maps) $F=\left\{f_{n}\right\}_{n=0}^{\infty}$.

Definition 2.2 Let $(X, d)$ be a metric space and $f_{n}: X \rightarrow X$ be a sequence of maps, $n=0,1,2, \ldots$. The time varying map $F=\left\{f_{n}\right\}_{n=0}^{\infty}$ is said to be expansive if there exists a constant $c>0$ (called an expansive constant) such that for any $x, y \in X, x \neq y, d\left(F_{n}(x), F_{n}(y)\right)>c$ for some $n \geq 0$. Equivalently, if for $x, y \in X$, $d\left(F_{n}(x), F_{n}(y)\right) \leq c$ for all $n \geq 0$ then $x=y$.

Remark 2.1 If in the above definition $f_{n}=f$ for all $n \geq 0$, where $f: X \rightarrow X$ is continuous, then expansiveness of time varying map $F=\left\{f_{n}\right\}_{n=0}^{\infty}$ on $X$ is equivalent to positive-expansiveness of $f$ on $X$ [9].

Remark 2.2 Note that expansiveness of a time variant map $F$ is independent of choice of metric if $X$ is compact. Let $d_{1}$ and $d_{2}$ be two equivalent metrics on a compact space $X$. Suppose $F$ is expansive on $\left(X, d_{1}\right)$ with expansive constant $\varepsilon>0$. Since $d_{1}$ is equivalent to $d_{2}$, there exists an $\varepsilon_{1}>0$ such that for any $x \in X, N_{d_{2}}\left(x, \varepsilon_{1}\right) \subset$ $N_{d_{1}}(x, \varepsilon)$, where $N_{d_{i}}(z, \delta)$ denotes open ball centred at $z$ in $X$ of radius $\delta$ under metric $d_{i}, i=1,2$. Since $X$ is compact, $\varepsilon_{1}$ depends only on $\varepsilon$ not on $x$. Let $x \neq y$. Since $F$ is expansive in $\left(X, d_{1}\right)$ with expansive constant $\varepsilon>0, F_{n}(y) \notin N_{d_{1}}\left(F_{n}(x), \varepsilon\right)$ for some $n \geq 0$. Now since $N_{d_{2}}\left(F_{n}(x), \varepsilon_{1}\right) \subset N_{d_{1}}\left(F_{n}(x), \varepsilon\right), F_{n}(y) \notin N_{d_{2}}\left(F_{n}(x), \varepsilon_{1}\right)$. Thus $F$ is expansive on $\left(X, d_{2}\right)$ with expansive constant $\varepsilon_{1}$.

Example 2.1 Consider the time varying map $F=\left\{f_{n}\right\}_{n=0}^{\infty}$ on the real line $\mathbb{R}$ defined by $f_{n}(x)=(n+1) x$, for $x \in \mathbb{R}$ and $n \geq 0$.

Choose $c>0$. Then for $x, y \in \mathbb{R}, x \neq y$, there exists $n \geq 0$ such that $\mid F_{n}(x)-$ $F_{n}(y)|=(n+1) !| x-y \mid>c$. Thus $F$ is expansive with expansive constant $c$.

Definition 2.3 If $h: X \rightarrow Y$ is a homeomorphism, $h$ is uniformly continuous on $X$ and $h^{-1}$ is uniformly continuous on $Y$, then $h$ is said to be a uniform homeomorphism. 
Definition 2.4 Let $\left(X, d_{1}\right)$ and $\left(Y, d_{2}\right)$ be two metric spaces. Let $F=\left\{f_{n}\right\}_{n=0}^{\infty}$ and $G=\left\{g_{n}\right\}_{n=0}^{\infty}$ be time varying maps on $X$ and $Y$ respectively. If there is a homeomorphism $h: X \rightarrow Y$ such that $h \circ f_{n}=g_{n} \circ h$ for all $n=0,1,2, \ldots$ then $F$ and $G$ are said to be conjugate (with respect to the map $h$ ) or $h$-conjugate. In particular, if $h: X \rightarrow Y$ is a uniform homeomorphism then $F$ and $G$ are said to be uniformly conjugate or uniformly $h$-conjugate.

For example, if $F=\left\{x^{n+1}\right\}_{n=0}^{\infty}$ on $[0,1], G=\left\{2((x+1) / 2)^{n+1}-1\right\}_{n=0}^{\infty}$ on $[-1,1]$ then $F$ is uniformly $h$-conjugate to $G$, where $h:[0,1] \rightarrow[-1,1]$ is defined by $h(x)=2 x-1$.

Theorem 2.1 Let $\left(X, d_{1}\right)$ and $\left(Y, d_{2}\right)$ be metric spaces. Let $F=\left\{f_{n}\right\}_{n=0}^{\infty}$ and $G=$ $\left\{g_{n}\right\}_{n=0}^{\infty}$ be time varying maps on $X$ and $Y$ respectively such that $F$ is uniformly conjugate to $G$. If $F$ is expansive on $X$ then $G$ is expansive on $Y$.

Proof Let $\varepsilon>0$ be an expansive constant for $F$. Since $F$ is uniformly conjugate to $G$, there exists a uniform homeomorphism $h: X \rightarrow Y$ such that $h \circ f_{n}=g_{n} \circ h$ for all $n \geq 0$. i.e. $f_{n} \circ h^{-1}=h^{-1} \circ g_{n}$ for all $n \geq 0$ which implies $F_{n} \circ h^{-1}=h^{-1} \circ G_{n}$ for all $n \geq 0$. Now, $h$ being a uniform homeomorphism, $h^{-1}$ is uniformly continuous therefore for $\varepsilon>0$ there exists a $\delta>0$ such that for $x, y \in Y, d_{2}(x, y)<\delta$ implies $d_{1}\left(h^{-1}(x), h^{-1}(y)\right)<\varepsilon$. Let $x, y \in Y$. If for all $n \geq 0 d_{2}\left(G_{n}(x), G_{n}(y)\right)<\delta$ then $d_{1}\left(h^{-1}\left(G_{n}(x)\right), h^{-1}\left(G_{n}(y)\right)\right)<\varepsilon$ for all $n \geq 0$. i.e. $d_{1}\left(F_{n}\left(h^{-1}(x)\right), F_{n}\left(h^{-1}(y)\right)\right)<$ $\varepsilon$ for all $n \geq 0$. Since $F$ is expansive with expansive constant $\varepsilon$, we get $h^{-1}(x)=$ $h^{-1}(y)$ which implies $x=y$. Thus $G$ is expansive with expansive constant $\delta$.

Corollary 2.1 Let $\left(X, d_{1}\right)$ be a compact metric space, $\left(Y, d_{2}\right)$ be a metric space, $F=\left\{f_{n}\right\}_{n=0}^{\infty}$ be a time varying map on $X$ and $h: X \rightarrow Y$ is a homeomorphism. If $F$ is expansive on $X$ then $G=h \circ F \circ h^{-1}=\left\{h \circ f_{n} \circ h^{-1}\right\}_{n=0}^{\infty}$ is expansive on $Y$.

Theorem 2.2 Let $(X, d)$ be a compact metric space, $\left\{f_{n}\right\}_{n=0}^{\infty}$ be an equicontinuous family of self maps on $X$ and $k$ be a positive integer. Then time varying map $F=$ $\left\{f_{n}\right\}_{n=0}^{\infty}$ is expansive if and only if $F^{k}$ is expansive.

Proof Let $e>0$ be an expansive constant for $F$. Since $\left\{f_{n}\right\}_{n=0}^{\infty}$ is equicontinuous family, for any $n>0$ and $n k+1 \leq j \leq(n+1) k, F_{[n k+1, j]}$ is uniformly continuous on $X$ and therefore there exists a $\delta_{j}>0$ such that

$$
d(x, y)<\delta_{j} \Rightarrow d\left(F_{[n k+1, j]}(x), F_{[n k+1, j]}(y)\right)<e .
$$

Note that due to equicontinuity of $\left\{f_{n}\right\}_{n=0}^{\infty}, \delta_{j}$ does not depend on $n$. Take $\delta=$ $\min \left\{\delta_{j}: n k+1 \leq j \leq(n+1) k\right\}$. Then for any $n \geq 0$,

$$
d(x, y)<\delta \Rightarrow d\left(F_{[n k+1, j]}(x), F_{[n k+1, j]}(y)\right)<e .
$$

Now $F^{k}=\left\{g_{n}\right\}_{n=0}^{\infty}$, where $g_{n}=F_{[(n-1) k+1, n k]}$ and $G_{n}=g_{n} \circ \cdots \circ g_{1} \circ g_{0}$. It is easy to see that $G_{n}=F_{n k}$. Note that for any $j \geq 0$ there exists $n \geq 0$ such that $n k \leq j \leq(n+1) k$. Now for any $n \geq 0$ and $n k \leq j \leq(n+1) k$, 


$$
\begin{aligned}
d\left(G_{n}(x), G_{n}(y)\right)<\delta & \Rightarrow d\left(F_{n k}(x), F_{n k}(y)\right)<\delta \\
& \Rightarrow d\left(F_{[n k+1, j]}\left(F_{n k}(x)\right), F_{[n k+1, j]}\left(F_{n k}(y)\right)\right)<e \\
& \Rightarrow d\left(F_{j}(x), F_{j}(y)\right)<e .
\end{aligned}
$$

Since $e$ is an expansive constant for $F, x=y$ and hence $\delta$ is an expansive constant for $F^{k}$.

Conversely, if $F^{k}$ is expansive with an expansive constant $\varepsilon$ then for any $x, y \in$ $X, \quad x \neq y$, there exists $n \geq 0$ such that $d\left(G_{n}(x), G_{n}(y)\right)>\varepsilon$ which implies $d\left(F_{n k}(x), F_{n k}(y)\right)>\varepsilon$ proving that $\varepsilon$ is an expansive constant for $F$.

Example 2.2 Let $N$ be any positive integer. Consider the time varying map $F=$ $\left\{f_{n}\right\}_{n=0}^{\infty}$ on the unit circle $S^{1}$ defined by

$$
f_{n}(z)= \begin{cases}z^{k+1} & 0 \leq n=2 k \leq 2 N \\ z^{\frac{1}{k+2}} & 1 \leq n=2 k+1<2 N \\ z & n>2 N .\end{cases}
$$

for any $z \in S^{1}$,where $z^{\frac{1}{m}}=\exp \left\{\frac{i}{m} \operatorname{Arg}(z)\right\}$, in which $\operatorname{Arg}(z)$ is the principal argument of $z$.

Note that $\left\{f_{n}\right\}_{n=0}^{\infty}$ is equicontinuous family of maps on compact space $S^{1}$ and $F^{2}=\left\{g_{n}\right\}_{n=0}^{\infty}$, where each $g_{n}=F_{[2 n-1,2 n]}$ is the identity map. Since $F^{2}$ is not expansive, by Theorem $2.2, F$ is not expansive.

Example 2.3 Consider the time varying map $F=\left\{f_{n}\right\}_{n=0}^{\infty}$ on the unit circle $S^{1}$ defined by

$$
f_{n}(z)= \begin{cases}z^{\frac{n}{2}+1} & \text { if } n \text { is even } \\ z^{\frac{2}{n+1}} & \text { if } n \text { is odd }\end{cases}
$$

for any $z \in S^{1}$, where $z \in S^{1}, z^{\frac{1}{m}}=\exp \left\{\frac{i}{m} \operatorname{Arg}(z)\right\}$, in which $\operatorname{Arg}(z)$ is the principal argument of $z$.

Note that

$$
F_{n}(z)= \begin{cases}z^{\frac{n}{2}+1} & \text { if } n \text { is even } \\ z & \text { if } n \text { is odd }\end{cases}
$$

for any $z \in S^{1}$ and therefore $F$ is expansive. Observe that $F^{2}=\left\{g_{n}\right\}_{n=0}^{\infty}$, where each $g_{n}=F_{[2 n-1,2 n]}$ is the identity map, therefore $F^{2}$ is not expansive. Note that $\left\{f_{n}\right\}$ is not equicontinuous family on $S^{1}$. Thus 'equicontinuity' in the hypothesis of Theorem 2.2 is necessary.

Definition 2.5 Let $(X, d)$ be a metric space, $F=\left\{f_{n}\right\}_{n=0}^{\infty}$ be a time varying map on $X$ and $Y$ be a subset of $X$. Then $Y$ is said to be invariant under $F$ if $f_{n}(Y) \subset Y$ for all $n \geq 0$, equivalently $F_{n}(Y) \subset Y$ for all $n \geq 0$. 
Theorem 2.3 Let $(X, d)$ be a metric space, $F=\left\{f_{n}\right\}_{n=0}^{\infty}$ be a time varying map which is expansive on $X$ and $Y$ be an invariant subset of $X$, then restriction of $F$ to $Y$, defined by $F \mid Y=\left\{f_{n} \mid Y\right\}$ is expansive.

Proof Let $\varepsilon>0$ be an expansive constant for $F$ on $X$. Let $x \neq y, x, y \in Y$ then $x, y \in X$ also, therefore there exists $n \geq 0$ such that $d\left(F_{n}(x), F_{n}(y)\right)>\varepsilon$. Since $Y$ is invariant under $F, F_{n}(x), F_{n}(Y) \in Y$. Hence $F \mid Y$ is also expansive with expansive constant $\varepsilon$.

Theorem 2.4 Let $\left(X, d_{1}\right)$ and $\left(Y, d_{2}\right)$ be metric space and $F=\left\{f_{n}\right\}_{n=0}^{\infty}, \quad G=$ $\left\{g_{n}\right\}_{n=0}^{\infty}$ be expansive time varying maps on $X$ and $Y$ respectively. Then under metric $d$ on $X \times Y$ defined by

$$
d\left(\left(x_{1}, y_{1}\right),\left(x_{2}, y_{2}\right)\right)=\max \left\{d_{1}\left(x_{1}, x_{2}\right), d_{2}\left(y_{1}, y_{2}\right)\right\} ; \quad\left(x_{1}, y_{1}\right),\left(x_{2}, y_{2}\right) \in X \times Y,
$$

time varying map $F \times G=\left\{f_{n} \times g_{n}\right\}_{n=0}^{\infty}$ is expansive on $X \times Y$. Hence every finite direct product of expansive time varying maps is expansive.

Proof Note that for any $n \geq 0$,

$$
(F \times G)_{n}(x, y)=\left(F_{n}(x), G_{n}(y)\right), \quad(x, y) \in X \times Y .
$$

Let $\varepsilon_{1}$ and $\varepsilon_{2}$ be expansive constants for $F$ and $G$ respectively. Let $\varepsilon=\min \left\{\varepsilon_{1}, \varepsilon_{2}\right\}$ and $\left(x_{1}, y_{1}\right),\left(x_{2}, y_{2}\right) \in X \times Y$.

If for all $n \geq 0, d\left((F \times G)_{n}\left(x_{1}, y_{1}\right),(F \times G)_{n}\left(x_{2}, y_{2}\right)\right)<\varepsilon$ then

$$
d\left(\left(F_{n}\left(x_{1}\right), G_{n}\left(y_{1}\right)\right),\left(F_{n}\left(x_{2}\right), G_{n}\left(y_{2}\right)\right)\right)<\varepsilon
$$

which implies

$$
\max \left\{d_{1}\left(F_{n}\left(x_{1}\right), F_{n}\left(x_{2}\right)\right), d_{2}\left(G_{n}\left(y_{1}\right), G_{n}\left(y_{2}\right)\right)\right\}<\varepsilon
$$

Hence $d_{1}\left(F_{n}\left(x_{1}\right), F_{n}\left(x_{2}\right)\right)<\varepsilon \leq \varepsilon_{1}$ and $d_{2}\left(G_{n}\left(y_{1}\right), G_{n}\left(y_{2}\right)\right)<\varepsilon \leq \varepsilon_{2}$, for all $n \geq 0$ which by expansiveness of $F$ and $G$ implies $x_{1}=x_{2}$ and $y_{1}=y_{2}$. i.e. $\left(x_{1}, y_{1}\right)=\left(x_{2}, y_{2}\right)$. Hence $F \times G$ is expansive with expansive constant $\varepsilon$.

\section{Shadowing or pseudo orbit tracing property (P.O.T.P.)}

Definition 3.1 Let $(X, d)$ be a metric space and $F=\left\{f_{n}\right\}_{n=0}^{\infty}$ be a time varying map on $X$. For $\delta>0$, the sequence $\left\{x_{n}\right\}_{n=0}^{\infty}$ in $X$ is said to be a $\delta$-pseudo orbit of $F$ if $d\left(f_{n+1}\left(x_{n}\right), x_{n+1}\right)<\delta$ for all $n=0,1,2, \ldots$

For given $\varepsilon>0$, a $\delta$-pseudo orbit $\left\{x_{n}\right\}_{n=0}^{\infty}$ is said to be $\varepsilon$-traced by $y \in X$ if $d\left(F_{n}(y), x_{n}\right)<\varepsilon$ for all $n=0,1,2, \ldots$

The time varying map $F$ is said to have shadowing property or pseudo orbit tracing property (P.O.T.P) if for every $\varepsilon>0$, there exists a $\delta>0$ such that every $\delta$-pseudo orbit is $\varepsilon$ - traced by some point of $X$. 
Remark 3.1 If in the above definition $f_{n}=f$ for all $n \geq 0$, where $f: X \rightarrow X$ is continuous, then P.O.T.P of time varying map $F=\left\{f_{n}\right\}_{n=0}^{\infty}$ on $X$ is equivalent to P.O.T.P. of $f$ on $X$ [2].

Remark 3.2 Note that shadowing property is independent of metric if $X$ is compact. Let $d_{1}$ and $d_{2}$ be two equivalent metrics on a compact space $X$. Suppose $F$ has P.O.T.P. in $\left(X, d_{1}\right)$. Let $\varepsilon>0$ be given. Since $d_{1}$ is equivalent to $d_{2}$, there exists an $\varepsilon_{1}>0$ such that for any $x \in X, N_{d_{1}}\left(x, \varepsilon_{1}\right) \subset N_{d_{2}}(x, \varepsilon)$. Since $X$ is compact $\varepsilon_{1}$ only depends on $\varepsilon$ but not on point $x$. Since $F$ has P.O.T.P. in $\left(X, d_{1}\right)$, for this $\varepsilon_{1}$, we get a $\delta_{1}>0$ such that any $\delta_{1}$-pseudo-orbit is $\varepsilon_{1}$-traced by some point.

Further, since $d_{1}$ and $d_{2}$ are equivalent, for this $\delta_{1}$, we get a $\delta>0$ such that for any $x \in X, N_{d_{2}}(x, \delta) \subset N_{d_{1}}\left(x, \delta_{1}\right)$. Now let $\left\{x_{n}\right\}_{n=0}^{\infty}$ be a $\delta$-pseudo-orbit of $F$ in $\left(X, d_{2}\right)$. Thus $f_{n+1}\left(x_{n}\right) \in N_{d_{2}}\left(x_{n+1}, \delta\right) \subset N_{d_{1}}\left(x_{n+1}, \delta_{1}\right)$. Hence $\left\{x_{n}\right\}_{n=0}^{\infty}$ is a $\delta_{1}$-pseudo orbit of $F$ in $\left(X, d_{1}\right)$, therefore there exists a $y \in X$ which $\varepsilon_{1}$-traces $\left\{x_{n}\right\}_{n=0}^{\infty}$. Thus $F_{n}(y) \in N_{d_{1}}\left(x_{n}, \varepsilon_{1}\right) \subset N_{d_{2}}\left(x_{n}, \varepsilon\right)$ which implies $\left\{x_{n}\right\}_{n=0}^{\infty}$ is $\varepsilon$-traced by $y$ in $\left(X, d_{2}\right)$. Hence $F$ has P.O.T.P in $\left(X, d_{2}\right)$.

Theorem 3.1 Let $\left(X, d_{1}\right)$ and $\left(Y, d_{2}\right)$ be metric spaces. Let $F=\left\{f_{n}\right\}_{n=0}^{\infty}$ and $G=$ $\left\{g_{n}\right\}_{n=0}^{\infty}$ be time varying maps on $X$ and $Y$ respectively such that $F$ is uniformly conjugate to $G$. If $F$ has P.O.T.P. then $G$ has P.O.T.P.

Proof Let $\varepsilon>0$ be given. Since $F$ is uniformly conjugate to $G$, there exists a uniform homeomorphism $h: X \rightarrow Y$ such that $h \circ f_{n}=g_{n} \circ h$. i.e. $f_{n} \circ h^{-1}=h^{-1} \circ$ $g_{n}$ for all $n \geq 0$. Now $h$ is uniformly continuous being uniform homeomorphism, therefore there exists an $\varepsilon_{0}>0$ such that $d_{1}(x, y)<\varepsilon_{0}$ implies $d_{2}(h(x), h(y))<\varepsilon$. Since $F$ has P.O.T.P. there exists a $\delta_{0}>0$ such that any $\delta_{0}$-pseudo orbit of $F$ is $\varepsilon_{0}$-traced by $F$ orbit of some point of $X$. Since $h$ being a uniform homeomorphism, $h^{-1}$ is uniformly continuous map, therefore for $\delta_{0}>0$ there exists a $\delta>0$ such that $d_{2}(x, y)<\delta$ implies $d_{1}\left(h^{-1}(x), h^{-1}(y)\right)<\delta_{0}$. Let $\left\{x_{n}\right\}_{n=0}^{\infty}$ be a $\delta$-pseudo orbit for $G$. i.e. $d_{2}\left(g_{n+1}\left(x_{n}\right), x_{n+1}\right)<\delta$. i.e. $d_{1}\left(h^{-1}\left(g_{n+1}\left(x_{n}\right)\right), h^{-1}\left(x_{n+1}\right)\right)<\delta_{0}$. i.e. $d_{1}\left(f_{n+1}\left(h^{-1}\left(x_{n}\right)\right), h^{-1}\left(x_{n+1}\right)\right)<\delta_{0}$ which implies $\left\{h^{-1}\left(x_{n}\right)\right\}_{n=0}^{\infty}$ is a $\delta_{0}$-pseudo orbit for $F$. Thus there exists a $y \in X$ such that $d_{1}\left(F_{n}(y), h^{-1}\left(x_{n}\right)\right)<\varepsilon_{0}$ and hence $d_{2}\left(h\left(F_{n}(y)\right), x_{n}\right)<\varepsilon$. Now for all $n \geq 0$,

$$
\begin{aligned}
h \circ F_{n}= & h \circ f_{n} \circ f_{n-1} \circ \cdots f_{2} \circ f_{1} \circ f_{0} \\
= & g_{n} \circ h \circ f_{n-1} \circ \cdots f_{2} \circ f_{1} \circ f_{0} \\
& \vdots \\
= & g_{n} \circ g_{n-1} \circ \cdots g_{2} \circ g_{1} \circ g_{0} \circ h \\
= & G_{n} \circ h
\end{aligned}
$$

implies $d_{2}\left(G_{n}(h(y)), x_{n}\right)<\varepsilon$. i.e. $\left\{x_{n}\right\}_{n=0}^{\infty}$ is $\varepsilon$-traced by $h(y) \in Y$. Thus $G$ has P.O.T.P.

Theorem 3.2 Let $\left(X, d_{1}\right)$ and $\left(Y, d_{2}\right)$ be metric spaces and $F=\left\{f_{n}\right\}_{n=0}^{\infty}, \quad G=$ $\left\{g_{n}\right\}_{n=0}^{\infty}$ be time varying maps on $X$ and $Y$ respectively. Define metric $d$ on $X \times Y$ by

$$
d\left(\left(x_{1}, y_{1}\right),\left(x_{2}, y_{2}\right)\right)=\max \left\{d_{1}\left(x_{1}, x_{2}\right), d_{2}\left(y_{1}, y_{2}\right)\right\}, \quad\left(x_{1}, y_{1}\right),\left(x_{2}, y_{2}\right) \in X \times Y .
$$


If $F$ and $G$ have P.O.T.P. then the time varying map $F \times G=\left\{f_{n} \times g_{n}\right\}_{n=0}^{\infty}$ has P.O.T.P. in $X \times Y$. Hence every finite direct product of time varying maps having P.O.T.P., has P.O.T.P.

Proof Note that for any $n \geq 0$,

$$
(F \times G)_{n}(x, y)=\left(F_{n}(x), G_{n}(y)\right) \quad(x, y) \in X \times Y .
$$

Let $\varepsilon>0$ be given. Then there exists a $\delta_{1}>0$ and a $\delta_{2}>0$ such that every $\delta_{1}$-pseudo orbit of $F$ and $\delta_{2}$-pseudo orbit of $G$ can be $\varepsilon$-traced by some $F$-orbit and $G$-orbit respectively. Let $\delta=\min \left\{\delta_{1}, \delta_{2}\right\}$ and $\left\{\left(x_{i}, y_{i}\right)\right\}_{i=0}^{\infty}$ be a $\delta$ pseudo orbit of $F \times G$. Then $d\left(\left(f_{i+1} \times g_{i+1}\right)\left(x_{i}, y_{i}\right),\left(x_{i+1}, y_{i+1}\right)\right)<\delta$. i.e. $d\left(\left(f_{i+1}\left(x_{i}\right), g_{i+1}\left(y_{i}\right)\right),\left(x_{i+1}, y_{i+1}\right)\right)<\delta$ which by definition of $d$ implies $d_{1}\left(f_{i+1}\left(x_{i}\right), x_{i+1}\right)<\delta \leq \delta_{1}$ and $d_{2}\left(g_{i+1}\left(y_{i}\right), y_{i+1}\right)<\delta \leq \delta_{2}$. Hence there exist $x \in X$ and $y \in Y$ such that $d_{1}\left(F_{i}(x), x_{i}\right)<\varepsilon$ and $d_{2}\left(G_{i}(y), y_{i}\right)<\varepsilon$. Hence $d\left(\left(F_{i}(x), G_{i}(y)\right),\left(x_{i}, y_{i}\right)\right)<\varepsilon$. i.e. $d\left((F \times G)_{i}(x, y),\left(x_{i}, y_{i}\right)\right)<\varepsilon$ which implies $\left\{\left(x_{i}, y_{i}\right)\right\}_{i=0}^{\infty}$ is $\varepsilon$-traced by $(x, y) \in X \times Y$. Hence any $\delta$-pseudo orbit of $F \times G$ can be $\varepsilon$-traced by some point of $X \times Y$. Thus $F \times G$ also has P.O.T.P. By induction, we get that every finite direct product of time varying maps having P.O.T.P. has P.O.T.P.

Theorem 3.3 Let $F=\left\{f_{n}\right\}_{n=0}^{\infty}$ be the time varying map on a metric space $(X, d)$. If $F$ has P.O.T.P. then $F^{k}$ has P.O.T.P. for every $k>0$.

Proof If $k=1$ nothing to prove. Suppose $k \geq 2$. Let $\varepsilon>0$ be given. Since $F$ has P.O.T.P., therefore there exists a $\delta>0$ such that every $\delta$-pseudo orbit of $F$ is $\varepsilon$-traced by some point of $X$. Let $\left\{y_{i}\right\}_{i=0}^{\infty}$ be a $\delta$-pseudo orbit of $F^{k}$. Then $d\left(g_{n+1}\left(y_{n}\right), y_{n+1}\right)<\delta$ for all $n \geq 0$, where $g_{n}=F_{[(n-1) k+1, n k]}$, i.e $d\left(F_{[n k+1,(n+1) k]}\left(y_{n}\right), y_{n+1}\right)<\delta$, for all $n \geq 0$.

For $0 \leq j<k$ and $n \geq 0$ put $x_{n k+j}=F_{[n k+1, n k+j]}\left(y_{n}\right)$.

Claim $\left\{x_{i}\right\}_{n=0}^{\infty}$ is a $\delta$-pseudo orbit for $F$.

i.e. to show : $d\left(f_{n k+j+1}\left(x_{n k+j}\right), x_{n k+j+1}\right)<\delta$, for all $n \geq 0$ and for all $j, 0 \leq$ $j<k$.

Choose any $n \geq 0$. Now for any $j, 0 \leq j \leq k-2$,

$$
f_{n k+j+1}\left(x_{n k+j}\right)=f_{n k+j+1}\left(F_{[n k+1, n k+j]}\left(y_{n}\right)\right)=F_{[n k+1, n k+j+1]}\left(y_{n}\right)=x_{n k+j+1} \text {. }
$$

Thus $d\left(f_{n k+j+1}\left(x_{n k+j}\right), x_{n k+j+1}\right)=0<\delta$ for all $j, 0 \leq j \leq k-2$.

Now for $j=k-1$,

$$
\begin{aligned}
d\left(f_{n k+k}\left(x_{n k+k-1}\right), x_{n k+k}\right) & =d\left(f_{n k+k}\left(F_{[n k+1, n k+k-1]}\left(y_{n}\right)\right), x_{(n+1) k}\right) \\
& =d\left(F_{[n k+1,(n+1) k]}\left(y_{n}\right), y_{n+1}\right) \\
& <\delta
\end{aligned}
$$

Hence the claim. By P.O.T.P. of $F,\left\{x_{i}\right\}_{i=0}^{\infty}$ is $\varepsilon$-traced by some $y \in X$. i.e. $d\left(F_{i}(y), x_{i}\right)<\varepsilon$, for all $i \geq 0$. In particular for $i=k n, d\left(F_{k n}(y), x_{k n}\right)<\varepsilon$. Thus $d\left(G_{n}(y), y_{n}\right)<\varepsilon$, where $G_{n}=g_{n} \circ \cdots \circ g_{1} \circ g_{0}=F_{n k}$. Thus $F^{k}=\left\{g_{n}\right\}_{n=0}^{\infty}$ has P.O.T.P. 
Remark 3.3 For time varying map given in Example 2.2, $F^{2}=\left\{g_{n}\right\}_{n=0}^{\infty}$, where each $g_{n}$ is the identity map. Now since $F^{2}$ does not have P.O.T.P., by above theorem $F$ does not have P.O.T.P.

Lemma 3.1 Let $(X, d)$ be a compact metric space, $F=\left\{f_{n}\right\}_{n=0}^{\infty}$ be a time varying map on $X$ (where each $f_{n}$ is continuous on $X$ ) and $N$ be a natural number. Then for every $\varepsilon>0$ there exists $\delta>0$ such that each $\delta$-finite pseudo orbit $\left\{x_{i}: 0 \leq i \leq N\right\}$ satisfies $d\left(F_{i}\left(x_{0}\right), x_{i}\right)<\varepsilon$, for $0 \leq i \leq N$.

Proof Let $\varepsilon>0$ be given. For $N=1$, take $\delta=\varepsilon$. Here $d\left(F_{0}\left(x_{0}\right), x_{0}\right)=d\left(x_{0}, x_{0}\right)=$ $0<\varepsilon$ and since $\left\{x_{i}: 0 \leq i \leq 1\right\}$ is an $\varepsilon$-pseudo orbit, we have $d\left(f_{1}\left(x_{0}\right), x_{1}\right)<\varepsilon$. i.e. $d\left(F_{1}\left(x_{0}\right), x_{1}\right)<\varepsilon$. Thus result holds for $N=1$. Suppose result holds for $N-1$. Since $f_{N}$ is uniformly continuous, for every $\varepsilon>0$ there exists $\varepsilon_{1}, 0<\varepsilon_{1}<\varepsilon$ such that $d(x, y)<\varepsilon_{1}$ implies $d\left(f_{N}(x), f_{N}(y)\right)<\frac{\varepsilon}{2} ; x, y \in X$. By our assumption there exists a $\delta_{1}, 0<\delta_{1}<\varepsilon$ such that each $\delta_{1}$-pseudo orbit $\left\{y_{i}: 0 \leq i \leq N-1\right\}$ is $\varepsilon_{1}$-traced by $y_{0} \in X$. We show that each $\frac{\delta_{1}}{2}$-pseudo orbit $\left\{x_{i}: 0 \leq i \leq \bar{N}\right\}$ is $\varepsilon$-traced by $x_{0} \in X$.

Since the $\frac{\delta_{1}}{2}$-pseudo orbit is a $\delta_{1}$-pseudo-orbit, the finite pseudo-orbit $\left\{x_{i}: 0 \leq i \leq\right.$ $N-1\}$ is $\varepsilon_{1}$-traced by the point $x_{0} \in X$. Hence $d\left(F_{i}\left(x_{0}\right), x_{i}\right)<\varepsilon_{1}<\varepsilon$, for $0 \leq i \leq$ $N-1$. In particular, $d\left(F_{N-1}\left(x_{0}\right), x_{N-1}\right)<\varepsilon_{1}$ and $\operatorname{so} d\left(f_{N}\left(F_{N-1}\left(x_{0}\right)\right), f_{N}\left(x_{N-1}\right)\right)<$ $\frac{\varepsilon}{2}$. i.e $d\left(F_{N}\left(x_{0}\right), f_{N}\left(x_{N-1}\right)\right)<\frac{\varepsilon}{2}$. Since $\left\{x_{i}: 0 \leq i \leq N\right\}$ is a $\frac{\delta_{1}}{2}$-pseudo orbit, we have $d\left(f_{N}\left(x_{N-1}\right), x_{N}\right)<\frac{\delta_{1}}{2}<\frac{\varepsilon}{2}$ and therefore

$$
\begin{aligned}
d\left(F_{N}\left(x_{0}\right), x_{N}\right) & \leq d\left(F_{N}\left(x_{0}\right), f_{N}\left(x_{N-1}\right)\right)+d\left(f_{N}\left(x_{N-1}\right), x_{N}\right) \\
& <\frac{\varepsilon}{2}+\frac{\varepsilon}{2} \\
& =\varepsilon .
\end{aligned}
$$

Hence result is true for $N$. Thus by principle of mathematical induction the result is true for any natural number $N$.

For any $m>0$, replacing $f_{i}$ by $f_{m+i}$ and $x_{i}$ by $x_{m+i}$ in Lemma 3.1 we get the following result:

Theorem 3.4 Let $(X, d)$ be a compact metric space and $F=\left\{f_{n}\right\}_{n=0}^{\infty}$ be a time varying map on $X$, where family $\left\{f_{n}\right\}$ is equicontinuous on $X$ and $N$ is a natural number. Then for every $\varepsilon>0$ there exists $a \delta>0$ such that for each $m \geq 0$ and each $\delta$-pseudo-orbit $\left\{x_{n}\right\}_{n=0}^{\infty}$, the finite $\delta$-pseudo orbit $\left\{x_{m+i}\right\}_{i=0}^{N}$ satisfies $d\left(F_{[m+1, m+i]}\left(x_{m}\right), x_{m+i}\right)<\varepsilon$ for all $i, 0 \leq i \leq N$.

Note that since $\left\{f_{n}\right\}$ is equicontinuous $\delta$ does not depend upon $m$.

Theorem 3.5 Let $(X, d)$ be a compact metric space, $F=\left\{f_{n}\right\}_{n=0}^{\infty}$ be a time varying map on $X$, where $\left\{f_{n}\right\}_{n=0}^{\infty}$ is equicontinuous on $X$ and $k>0$. If $F^{k}=\left\{g_{n}\right\}_{n=0}^{\infty}$, where $g_{n}=F_{[(n-1) k+1, n k]}$ for all $n \geq 0$, has P.O.T.P. then so does $F=\left\{f_{n}\right\}_{n=0}^{\infty}$.

Proof Let us first observe the following :

(I) Let $\varepsilon>0$ be given. Then for each $i \geq 0,\left\{F_{[i k+1, i k+j]}: 0 \leq j \leq k\right\}$ is equicontinuous being finite family of continuous functions on compact metric space $X$ therefore there exists an $\varepsilon_{1}, 0<\varepsilon_{1}<\varepsilon$ such that 


$$
d(x, y)<\varepsilon_{1} \text { implies } \max _{0 \leq j \leq k}\left\{d\left(F_{[i k+1, i k+j]}(x), F_{[i k+1, i k+j]}(y)\right)\right\}<\frac{\varepsilon}{2} .
$$

Note that $\varepsilon_{1}$ does not depend on $i$ as family $\left\{f_{n}\right\}$ is equicontinuous.

(II) Let $\varepsilon$ and $\varepsilon_{1}$ be as above. Then there exists a $\delta_{0}, 0<\delta_{0}<\varepsilon_{1}$ such that each finite $\delta_{0}$-pseudo orbit $\left\{x_{m+j}\right\}_{j=1}^{k}$ satisfies $d\left(F_{[m+1, m+j]}\left(x_{m}\right), x_{m+j}\right)<\frac{\varepsilon}{2}$ for all $j, 0 \leq j \leq k$ and for each $m \geq 0$ (by Theorem 3.4).

(III) Let $\varepsilon_{1}$ and $\delta_{0}$ be as above. Since $F^{k}$ has P.O.T.P., there exists a $\delta_{1}, 0<\delta_{1}<\delta_{0}$ such that any $\delta_{1}$-pseudo-orbit for $F^{k}$ is $\varepsilon_{1}$-traced by some point of $X$.

(IV) Let $\varepsilon_{1}$ and $\delta_{1}$ be as above. Then there exists a $\delta, 0<\delta<\delta_{1}$ such that each $\delta$-finite pseudo-orbit $\left\{z_{m+i}\right\}_{i=1}^{k}$ satisfies $d\left(F_{[m+1, m+i]}\left(z_{m}\right), z_{m+i}\right)<\delta_{1}$ for all $i$, $0 \leq i \leq k$ and for each $m \geq 0$ (by Theorem 3.4).

With these properties we prove that each $\delta$-pseudo orbit $\left\{y_{i}\right\}_{i=0}^{\infty}$ of $F$ is $\varepsilon$-traced by some point. Write $x_{i}=y_{i k}$ for $i=0,1,2, \ldots$ and fix $i$. Since $\left\{y_{i k+j}: 0 \leq j \leq\right.$ $k\}$ is a $\delta$-finite pseudo-orbit for $F$, by (IV) we have $d\left(F_{[i k+1, i k+j]}\left(y_{i k}\right), y_{i k+j}\right)<$ $\delta_{1}(0 \leq j \leq k)$ and especially if $j=k$ then $d\left(F_{[i k+1, i k+k]}\left(y_{i k}\right), y_{i k+k}\right)<\delta_{1}$ i.e. $d\left(g_{i+1}\left(x_{i}\right), x_{i+1}\right)<\delta_{1}$. i.e. $\left\{x_{i}\right\}_{i=0}^{\infty}$ is $\delta_{1}$-pseudo orbit for $F^{k}$. Hence by (III), there exists $y \in X$ with $d\left(G_{i}(y), x_{i}\right)<\varepsilon_{1}$, for $i=0,1,2, \ldots$, where $G_{i}=g_{i} \circ g_{i-1} \circ$ $\cdots \circ g_{1} \circ g_{0}=F_{i k}$. i.e. $d\left(F_{i k}(y), y_{i k}\right)<\varepsilon_{1}$, for $i=0,1,2, \ldots$ and hence from (I) $d\left(F_{[i k+1, i k+j]}\left(F_{i k}(y)\right), F_{[i k+1, i k+j]}\left(y_{i k}\right)\right)<\frac{\varepsilon}{2}$. i.e.

$$
d\left(F_{i k+j}(y), F_{[i k+1, i k+j]}\left(y_{i k}\right)\right)<\frac{\varepsilon}{2}, \quad o \leq j \leq k, \quad i \geq 0 .
$$

On the other hand, since $\left\{y_{i k+j}: 0 \leq j \leq k\right\}$ is a finite $\delta_{0}$-pseudo orbit of $F$ (as $\left\{y_{i}\right\}_{i=0}^{\infty}$ is a $\delta$-pseudo orbit and $\delta<\delta_{1}<\delta_{0}$, so $\left\{y_{i}\right\}_{i=0}^{\infty}$ is also $\delta_{0}$-pseudo orbit of $F$ ), from (II) it follows that for any $i \geq 0$, taking $m=i k$,

$$
d\left(F_{[i k+1, i k+j]}\left(y_{i k}\right), y_{i k+j}\right)<\frac{\varepsilon}{2},
$$

for $0 \leq j \leq k$. Therefore using Eqs. (1) and (2), we get

$$
\begin{aligned}
d\left(F_{i k+j}(y), y_{i k+j}\right) & \leq d\left(F_{i k+j}(y), F_{[i k+1, i k+j]}\left(y_{i k}\right)\right)+d\left(F_{[i k+1, i k+j]}\left(y_{i k}\right), y_{i k+j}\right) \\
& <\frac{\varepsilon}{2}+\frac{\varepsilon}{2} \\
& =\varepsilon,
\end{aligned}
$$

for $0 \leq j \leq k$. Since $i$ is arbitrary, we have $d\left(F_{n}(y), y_{n}\right)<\varepsilon$ for $n=0,1,2 \ldots$ and hence the $\delta$-pseudo orbit $\left\{y_{n}\right\}_{n=0}^{\infty}$ is $\varepsilon$-traced by $y$.

Example 3.1 Let $M>0$ and $F=\left\{f_{n}\right\}_{n=0}^{\infty}$ be a time varying map on [0,1] defined by

$$
f_{n}= \begin{cases}x^{2(k+1)} & 0 \leq n=2 k \leq 2 M \\ x^{\frac{1}{k+2}} & 1 \leq n=2 k+1<2 M \\ x^{2} & n>2 M .\end{cases}
$$


Here $F^{2}=\left\{g_{n}\right\}_{n=0}^{\infty}$, where $g_{n}(x)=x^{2}$ for all $n \geq 0$. Since map $x^{2}$ on $[0,1]$ has P.O.T.P. (by Lemma 4.1 in [6]), $F^{2}$ has P.O.T.P. By above theorem we get that $F$ has P.O.T.P.

\section{Topological stability of a time varying map on a compact metric space}

Let $(X, d)$ be a compact metric space, define standard bounded metric $d_{1}$ on $X$ by

$$
d_{1}(x, y)=\max \{d(x, y), 1\}, \quad x, y \in X .
$$

and $(\mathcal{C}(X), \eta)$ be the space of all continuous self maps on $X$, where metric $\eta$ is defined by

$$
\eta(f, g)=\sup _{x \in X} d_{1}(f(x), g(x)), \quad f, g \in \mathcal{C}(X)
$$

A time varying map is a countable subset of $\mathcal{C}(X)$. Let $S(X)$ be the collection of all time varying maps on $X$. We define a metric $\rho$ on $S(X)$ as follows:

For $F=\left\{f_{n}\right\}_{n=0}^{\infty}$ and $G=\left\{g_{n}\right\}_{n=0}^{\infty}$,

$$
\rho(F, G)=\sup _{n \geq 0} \eta\left(f_{n}, g_{n}\right)
$$

Definition 4.1 A time varying map $F$ is said to be topologically stable in $S(X)$, if for every $\varepsilon>0$ there exists a $0<\delta<1$ such that for a time varying map $G$ with $\rho(F, G)<\delta$ there is a continuous map $h$ so that for all $x \in X, d(h(x), x)<\varepsilon$ and $d\left(F_{n}(h(x)), G_{n}(x)\right)<\varepsilon$, for all $n \geq 0$.

Theorem 4.1 If a time varying map $F$ on a compact metric space $X$ is expansive and has P.O.T.P. then it is topologically stable in $S(X)$.

Proof Let $e>0$ be an expansive constant for time varying map $F=\left\{f_{n}\right\}_{n=0}^{\infty}$ and fix $0<\varepsilon<\frac{e}{3}$. Let $0<\delta<\min \left\{\frac{e}{3}, 1\right\}$ be such that every $\delta$-pseudo orbit of $F$ can be $\varepsilon$-traced by some $F$-orbit. By expansiveness of $F$, it follows that there exists a unique $x \in X$ which $\varepsilon$-traces a given $\delta$-pseudo orbit $\left\{x_{i}\right\}_{i=0}^{\infty}$. Indeed, let $y \in X$ be another $\varepsilon$-tracing point of $\left\{x_{i}\right\}_{i=0}^{\infty}$. Then we have $d\left(F_{n}(x), x_{n}\right)<\varepsilon$ and $d\left(F_{n}(y), x_{n}\right)<\varepsilon$ for all $n \geq 0$. Now

$$
d\left(F_{n}(x), F_{n}(y)\right) \leq d\left(F_{n}(x), x_{n}\right)+d\left(x_{n}, F_{n}(y)\right) \leq 2 \varepsilon<e,
$$

for all $n \geq 0$ and hence $x=y$.

Let $G=\left\{g_{n}\right\}_{n=0}^{\infty}$ be a time varying map on $X$ such that $\rho(F, G)<\delta$. Since $\delta<1, d\left(f_{n}(x), g_{n}(x)\right)<\delta$, for all $x \in X$ and for all $n \geq 0$. Let $x \in X$. Since $d\left(f_{n+1}\left(G_{n}(x)\right), G_{n+1}(x)\right)=d\left(f_{n+1}\left(G_{n}(x)\right), g_{n+1}\left(G_{n}(x)\right)\right)<\delta$, for all $n \geq 0$, $\left\{G_{n}(x)\right\}_{n=0}^{\infty}$ is a $\delta$ pseudo orbit for $F=\left\{f_{n}\right\}_{n=0}^{\infty}$. Thus there exists a unique point $h(x) \in X$ whose $F$-orbit $\varepsilon$-traces $\left\{G_{n}(x)\right\}_{n=0}^{\infty}$. This defines a map $h: X \rightarrow X$ with $d\left(F_{n}(h(x)), G_{n}(x)\right)<\varepsilon$ for $n \geq 0$ and $x \in X$. Letting $n=0$, we have $d(h(x), x)<\varepsilon$, for each $x \in X$. 
Finally, we show that $h$ is continuous. Let $\lambda>0$. Then we can choose $N>0$ such that,

$$
0 \leq n \leq N, \quad d\left(F_{n}(x), F_{n}(y)\right)<e \Rightarrow d(x, y)<\lambda
$$

Suppose this is false. Let $\alpha$ be an open cover of $X$ with diameter less than $e$. Then there exists an $\varepsilon>0$ such that for each $j \geq 0$, there exist $x_{j}, y_{j} \in X$ with $d\left(x_{j}, y_{j}\right)>\varepsilon$ and $A_{j, i} \in \alpha(0 \leq i \leq j)$ with $F_{i}\left(x_{j}\right), F_{i}\left(y_{j}\right) \in A_{j, i}, 0 \leq i \leq j$. Since $X$ is compact, there exist $x, y \in X$ such that $x_{j} \rightarrow x$ and $y_{j} \rightarrow y$. Note that $x \neq y$ as for each $j, d\left(x_{j}, y_{j}\right)>\varepsilon$. Consider the sets $A_{j, 0}$ for $j \geq 0$. Since $X$ is compact therefore $\alpha$ has finite subcover and hence infinitely many $A_{j, 0}$ coincide to some $A_{0} \in \alpha$. Thus $x_{j}, y_{j} \in A_{0}$ for infinitely many $j$ which implies $x, y \in \overline{A_{0}}$. Similarly for infinitely many $A_{j, n}$ they coincide to some $A_{n} \in \alpha$ and we have $F_{n}\left(x_{j}\right), F_{n}\left(y_{j}\right) \in A_{n}$ for infinitely many $j$. Thus $F_{n}$ being continuous, $F_{n}(x), F_{n}(y) \in \bar{A}_{n}$. Since $\operatorname{diam}\left(A_{n}\right)=$ $\operatorname{diam}\left(\overline{A_{n}}\right)<e$, we have $d\left(F_{n}(x), F_{n}(y)\right)<e$ for all $n \geq 0$, contradicting the fact that $F$ is expansive.

Since $\left\{G_{n}: 0 \leq n \leq N\right\}$ is uniformly equicontinuous on $X$, we can choose $\eta>0$ such that $d(x, y)<\eta$ implies $d\left(G_{n}(x), G_{n}(y)\right)<\frac{e}{3}$ for all $n, 0 \leq n \leq N$.

If $d(x, y)<\eta$ then for all $n, 0 \leq n \leq N$, we have

$$
\begin{aligned}
d\left(F_{n}(h(x)), F_{n}(h(y))\right) \leq & d\left(F_{n}(h(x)), G_{n}(x)\right)+d\left(G_{n}(x), G_{n}(y)\right) \\
& +d\left(G_{n}(y), F_{n}(h(y))\right) \leq \varepsilon+\frac{e}{3}+\varepsilon \\
< & e .
\end{aligned}
$$

Thus by Eq. (3), $d(h(x), h(y))<\lambda$. Therefore $d(x, y)<\eta$ implies $d(h(x), h(y))<\lambda$ proving continuity of $h$.

Remark 4.1 We generalize the concept of expansiveness for invertible time varying dynamical systems. If we consider the sequence $\left\{f_{n}\right\}_{n=0}^{\infty}$ as the time varying map $F$, where each $f_{n}$ is a homeomorphism on a compact metric space $X$ with $f_{0}$ as the identity map on $X$, we define for $n \geq 0$,

$$
F_{n}=f_{n} \circ f_{n-1} \circ \cdots \circ f_{1} \circ f_{0}
$$

and for $n=-m<0$,

$$
F_{n}=F_{(-m)}=\left(F_{m}\right)^{-1}=f_{0}^{-1} \circ f_{1}^{-1} \circ \cdots \circ f_{(m-1)}^{-1} \circ f_{m}^{-1} .
$$

For $x_{0} \in X$, orbit of $x_{0}$ is the sequence $\left\{x_{n}\right\}_{n=-\infty}^{\infty}$ where $x_{n+1}=f_{n+1}\left(x_{n}\right)$ for all $n \geq 0$ and $x_{n}=f_{(-n)}^{-1}\left(x_{n+1}\right)$ for all $n<0$.

For invertible time varying dynamical system $F$, we say that $F$ is expansive if there exists a constant $c>0$ (called an expansive constant) such that for any $x, y \in X, x \neq$ $y, d\left(F_{n}(x), F_{n}(y)\right)>c$ for some integer $n$.

Analogously we can define shadowing property and stability of invertible time varying dynamical systems and prove related results for such systems which are proved for classical dynamical systems given by a homeomorphism. 
Acknowledgments Authors thank the referee for drawing their attention to reference [1] and for some useful suggestions.

Open Access This article is distributed under the terms of the Creative Commons Attribution License which permits any use, distribution, and reproduction in any medium, provided the original author(s) and the source are credited.

\section{References}

1. Araújo, V.: Attractors and time averages for random maps. Ann. Inst. H. Poincaré Anal. Non Linéaire 17(3), 307-369 (2000)

2. Aoki, N., Hiraide, K.: Topological Theory of Dynamical Systems. North-Holland Publishing Co., Amsterdam (1994)

3. Bowen, R.: Entropy-expansive maps. Trans. Am. Math. Soc. 164, 323-331 (1972)

4. Bessa, M., Rocha, J.: A remark on the topological stability of symplectomorphisms. Appl. Math. Lett. 25, 163-165 (2012)

5. Bessa, M., Rocha, J.: Topological stability for conservative systems. J. Differ. Equ. 250(10), 3960-3966 (2011)

6. Chen, L., Li, S.: Shadowing property for inverse limit spaces. Proc. Am. Math. Soc. 115(2), 573-580 (1992)

7. Das, R.: Chaos of a sequence of maps in a metric $G$-space. Appl. Math. Sci. 136(6), 6769-6775 (2012)

8. Das, T., Lee, K., Richeson, D., Wiseman, J.: Spectral decomposition for topologically Anosov homeomorphisms on noncompact and non-metrizable spaces. Topology Appl. 160(1), 149-158 (2013)

9. Eisenberg, M.: Expansive transformation semigroups of endomorphisms. Fund. Math. 59, 313-321 (1966)

10. Hurley, M.: Fixed points of topologically stable flows. Trans. Am. Math. Soc. 294(2), 625-633 (1986)

11. Hurley, M.: Consequences of topological stability. J. Differ. Equ. 54(1), 60-72 (1984)

12. Hurley, M.: Combined structural and topological stability are equivalent to Axiom A and the strong transversality condition. Ergodic Theory Dyn. Syst. 4(1), 81-88 (1984)

13. Kato, H.: Continuum-wise expansive homeomorphisms. Can. J. Math. 45, 576-598 (1993)

14. Lee, K., Sakai, K.: Various shadowing properties and their equivalence. Discrete Contin. Dyn. Syst. 13(2), 533-540 (2005)

15. Morales, C.: A generalization of expansivity. Discrete Contin. Dyn. Syst. 32(1), 293-301 (2012)

16. Moriyasu, K.: The topological stability of diffeomorphisms. Nagoya Math. J. 123, 91-102 (1991)

17. Morales, C.: Measure-expansive systems. Preprint IMPA, D083 (2011)

18. Moriyasu, K., Sakai, K., Sumi, N.: Vector fields with topological stability. Trans. Am. Math. Soc. 353(8), 3391-3408 (2001)

19. Nitecki, Z.: On semi-stability for diffeomorphisms. Invent. Math. 14, 83-122 (1971)

20. Pilyugin, S.Y.: Shadowing in dynamical systems, Lecture Notes in Mathematics, vol. 1706. Springer, Berlin (1999)

21. Reddy, W.L.: Pointwise expansion homeomorphisms. J. Lond. Math. Soc. 2, 232-236 (1970)

22. Robinson, C.: Stability theorems and hyperbolicity in dynamical systems. Rocky Mt. J. Math. 7(3), 425-437 (1977)

23. Tian, C., Chen, G.: Chaos of a sequence of maps in a metric space. Chaos Solitons Fractals 28, 1067-1075 (2006)

24. Utz, W.R.: Unstable homeomorphisms. Proc. Am. Math. Soc. 1, 769-774 (1950)

25. Walters, P.: Anosov diffeomorphism are topologically stable. Topology 9, 71-78 (1970)

26. Walters, P.: On the pseudo orbit tracing property and its relationship to stability, Lecture Notes in Mathematics, vol. 668. Springer, Berlin, pp. 231-244 (1978) 